\title{
Editorial
}

\section{APPLIED PHARMACOECONOMICS IN PUBLIC HEALTH}

\section{Introduction}

Today's cost-sensitive healthcare ecosystem has created a competitive and demanding workplace for the clinicians. In the Indian healthcare scenario, the challenge is to provide healthcare to far-reaching places across inhospitable terrains amidst populace where literacy level is low. Availability of healthcare resources such as doctors, infrastructure and equipment is another huge necessity. The question is not of sufficiency; it is of equitable distribution of healthcare resources, which is a constant difficulty faced by our healthcare agencies. Present-day healthcare professionals also have to strive hard to provide quality patient care while assuring an efficient use of resources. Hospitals and physicians worldwide are increasingly facing problems in deciding which treatment is most effective medically and economically. There is an inherent dilemma of balancing cost and treatment. The decision is based on the most viable medical and economic options.

Pharmacoeconomic research is the process of identifying, measuring and comparing the costs, risks and benefits of programs, services or therapies and determining which alternative produces the best health outcome for the resource invested. The purpose of Pharmacoeconomics is to establish the relative worth of a product/service that can be used by decision-makers who have limited budgets.

Operationally, the field of Pharmacoeconomics deals with analysis and evaluation of outcomes (clinical, economic or humanistic), cost consequences and cost comparison (for example considering resource consumption); identification of alternatives; and decision-making considering limited (fixed) budget/resources. Healthcare costs or economic outcomes can be grouped into several categories: Direct medical, direct nonmedical, indirect nonmedical and intangible costs. It has also been defined as the description and analysis of the cost of drug therapy to healthcare systems and society. More specifically, it is of paramount importance to assess costs and consequences-the value of a pharmaceutical product or service-depends heavily on the perspective of the evaluation. Common perspectives include those of the patient, provider, payer, and society:

- Patient Perspective: Patient perspective is important because patients are the ultimate consumers of healthcare services. Costs from the perspective of patients are essentially what patients pay for a product or service-that is, the portion not covered by the insurance. Consequences, from a patient's perspective, are the clinical effects, both positive and negative, of a program or treatment alternative.

- Provider Perspective: Costs from the provider's perspective are the actual expense of providing a product or service, regardless of whether they are healthcare organizations or private-practice physicians. From this perspective, direct costs such as drugs, hospitalization, laboratory tests, supplies, and salaries of healthcare professionals can be identified, measured, and compared.

- Payer Perspective: Payers include insurance companies, employers, or the government. From this perspective, costs represent the charges for healthcare products and services allowed or reimbursed by the payer. The primary cost for a payer is of a direct nature. However, indirect costs are such costs as lost workdays (absenteeism), being at work but not feeling of health care to the payer.

- Societal Perspective: The perspective of society is the broadest of all perspectives because it is the only one that considers the benefit to society as a whole. Theoretically, all direct and indirect costs are included in an economic evaluation performed from a societal perspective.

- Researchers Perspective: A recent trend indicates that clinical trial is dependent on economic data collection. Some studies incorporate an element of modeling to adapt the findings of a trial to another trial. Several regulatory agencies are now evaluating the use of pharmacoeconomic parameters as a part of the drug regulatory process. A Pharmacoeconomics study from the researcher's perspective will usually consist of a decision analysis model containing an economic evaluation.

Methods of Pharmacoeconomics: The pharmacoeconomic methods of evaluation are various scientific tools, which can be separated into two distinct categories: economic and humanistic evaluation techniques. These methods have been used in a variety of fields and are economic tools such as CBA, CEA, cost utilization, etc. Simultaneously, humanistic 
outcome parameters such as quality of life, patient satisfaction, DALY, etc. should be monitored. These parameters will help in distributing the limited resources into much needed areas.

\section{Applications of Pharmacoeconomics in Hospitals}

Healthcare practitioners, regardless of practice setting, can benefit from applying the principles and methods of Pharmacoeconomics to their daily practice settings. Applied Pharmacoeconomics is putting pharmacoeconomic principles, methods, and theories into practice to quantify the value of pharmacy products and pharmaceutical care services used in real-world environments. One of the primary applications of Pharmacoeconomics in clinical practice, today, is to aid clinical decision making and policy making. Through the appropriate application of Pharmacoeconomics, practitioners and administrators can make better, more informed decisions regarding the products and services they provide. Complete pharmacotherapy decisions should contain assessments of three basic outcome areas whenever appropriate: Economic, Clinical and Humanistic outcomes (ECHO). Traditionally, most drug therapy decisions were based solely on the clinical outcomes (e.g., safety and efficacy) associated with a treatment alternative. Over the past 20 years, it has become quite popular also to include an assessment of the economic outcomes associated with a treatment alternative. The current trend is also to incorporate the humanistic outcomes associated with a treatment alternative, that is, to bring the patient back into this decision-making equation. This ECHO model for medical decision-making has become prevalent in the current healthcare settings. In today's healthcare environment, it is no longer appropriate to make drug-selection decisions based solely on acquisition costs.

\section{Clinical Pharmacy Service Evaluation}

The most recent application of pharmacoeconomic principles and methods has been for justifying the value of various healthcare services, particularly pharmacy services. When a specific service is competing for hospital or MCO resources, Pharmacoeconomics can provide the data necessary to show that the service maximizes due to the resources allocated by healthcare system administrators.

\section{Conclusion}

The principles and methods of Pharmacoeconomics provide the means to quantify the value of pharmacotherapy through balancing costs and outcomes. Providing quality care with minimal resources is the future. By understanding the principles, methods and application of Pharmacoeconomics, healthcare professionals will be prepared to make better, more informed decisions regarding the use of pharmaceutical products and services, that is, decisions that ultimately represent the best interests of the patient, the healthcare system and the society.

Shakti Kumar Gupta
Medical Superintendent
All India Institute of Medical Sciences, Dr. R P Centre for
Ophthalmic Sciences, New Delhi, India
Editor-in-Chief, IJRFHHA
Maj Gen Sunil Kant, VSM
Comdt, Command Hospital (EC)
Editor-in-Chief, IJRFHHA

